\title{
DETERMINAÇÃO DE COMPOSTOS DE MASSA MOLECULAR ALTA EM FOLHAS DE PLANTAS DA AMAZÔNIA
}

Denilson Soares de Siqueira, Alberto dos Santos Pereira e Francisco Radler de Aquino Neto*

Instituto de Química, Universidade Federal do Rio de Janeiro, CT, Bloco A, 21949-900 Rio de Janeiro - RJ

José Augusto Cabral e Carlos Alberto Cid Ferreira

Instituto Nacional de Pesquisa da Amazônia, Av. André Araújo. 2936, 69083-000 Manaus - AM

Bernd R. T. Simoneit

Petroleum and Environmental Geochemistry Group, College of Oceanic and Atmospheric Sciences, Oregon State University, Corvallis, Oregon 97331 - USA

Vladimir O. Elias

Analytical Solution, R. Prof. Saldanha, 115, 22461-220 Rio de Janeiro - RJ

\begin{abstract}
DETERMINATION OF HIGH MOLECULAR MASS COMPOUNDS FROM AMAZONIAN PLANT'S LEAVES. The fractIons of dichloromethane extracts of leaves from andiroba (Carapa guianensis - Meliaceae), caapi (Banisteriopsis caapi - Malpighiaceae), cocoa (Theobroma cacao - Sterculiaceae), Brazil nut (Bertholletia excelsa - Lecytidaceae), cupuaçu (Theobroma grandiflorum Sterculiaceae), marupá (Simaruba amara - Simaroubaceae) and rubber tree (Hevea brasiliensis - Euphorbiaceae), were analyzed by HT-HRGC and HT-HRGC-MS. Esters of homologous series of fatty acids and long chain alcohols, phytol, amyrines and tocopherols were characterized. The characterization of the compounds was based mainly in mass spectra data and in addition by usual spectrometric data $\left({ }^{1} \mathrm{H}\right.$ and ${ }^{13} \mathrm{C}$ NMR, IR).
\end{abstract}

Keywords: wax esters; phytyl esters; tocopherol esters.

\section{INTRODUÇÃO}

A utilização da Cromatografia Gasosa de Alta Resolução e Alta Temperatura (CGAR-AT) representa um enorme avanço para a análise de moléculas orgânicas não detectadas pela Cromatografia Gasosa de Alta Resolução (CGAR). Sendo a CGAR uma das técnicas de separação e identificação de compostos mais popularmente utilizada, não constitui surpresa que o número de aplicações tenha aumentado consideravelmente com o uso comercial das colunas de alta temperatura, conforme relatam Pereira e Aquino Neto ${ }^{1,2}$. Combinadas com a espectrometria de massas (CGAR-AT-EM) essas colunas têm sido utilizadas na análise de extratos de produtos naturais, buscando a caracterização de compostos de massa molecular alta ${ }^{3-5}$.

Os vegetais são organismos que possuem em sua constituição estruturas poliméricas como a cutina, suberina, celulose, lignina e outros biopolímeros. Em geral alguns órgãos (as folhas) são recobertos externamente por uma fina camada de cera, que desempenha grande importância biológica nas relações entre a planta e o meio ambiente em que esteja inserida. Por definição, ceras epidérmicas são compostos hidrofóbicos, da superfície das plantas, que são removidos por uma breve imersão em solvente orgânico, como clorofórmio ou hexano. Elas são misturas complexas de compostos de cadeias longas $\left(>\mathrm{C}_{18}\right)$, tais como ácidos graxos, hidrocarbonetos, álcoois, aldeídos, cetonas, ésteres, compostos alifáticos polinucleares e compostos fenólicos; compostos que podem apresentar massas moleculares altas ${ }^{6}$. É de se esperar essa composição predominante de moléculas maiores, pois as menores, com maior pressão de vapor, podem se volatilizar para o meio ambiente.

*e-mail: ladetec@iq.gov.br
Com o objetivo de avaliar a ocorrência desses compostos de massa molecular alta, foram estudados extratos de diclorometano de diversas plantas da Amazônia.

\section{PARTE EXPERIMENTAL}

\section{Obtenção dos extratos}

As folhas picotadas $(3,0 \mathrm{~g})$ foram colocadas em um balão de 100 $\mathrm{mL}$, onde se adicionou $15 \mathrm{~mL}$ de solvente (diclorometano) e submeteu-se a extração com ultra-som por 20 min. Após a extração, a solução foi filtrada e o filtrado concentrado até a secura sob fluxo de $\mathrm{N}_{2}$. Repetiu-se a extração por mais quatro vezes. Os extratos obtidos foram fracionados por Cromatografia em Camada Delgada (CCD), utilizando placas preparativas (placas de vidro 20 X $20 \mathrm{~cm}$, sílica com indicador fluorescente e espessura da camada de sílica de 2,0 mm). As placas foram eluídas, utilizando uma mistura de hexano e éter etílico (9:1) como eluente, até a frente do solvente atingir $18 \mathrm{~cm}$ de altura e, posteriormente, foram secadas e reveladas em câmara de ultravioleta. As regiões nas placas, que continham os constituintes, foram marcadas e a sílica correspondente foi retirada e extraída com hexano.

\section{Análise cromatográfica}

Os extratos (brutos e frações) foram analisados por CGAR-AT utilizando cromatógrafo HP 5890 e coluna capilar de vidro de $20 \mathrm{~m}$ x $0,25 \mathrm{~mm}$ e espessura de fase de $0,10 \mu \mathrm{m}$, com fase $20 \%$-fenil80\%-metilpolisiloxana (PS-090) e detector por ionização em chama. As condições cromatográficas: injetor tipo na coluna a frio ("cold on-column"), hidrogênio como gás carreador (1,5 $\mathrm{mL} / \mathrm{min})$; programação de temperatura de $40^{\circ}$ a $390{ }^{\circ} \mathrm{C}$ a uma taxa de $10{ }^{\circ} \mathrm{C} / \mathrm{min}$ e injeção de $0,5 \mu \mathrm{L}$ a uma concentração de 100 ppm de amostra. Para 
CGAR-AT-EM utilizou-se um instrumento Hewlet-Packard - HP5972 - com injetor do tipo na coluna a frio ("cold on-column"), impacto de elétrons a $70 \mathrm{eV}$, varredura linear no intervalo de 50 - $700 \mathrm{Da}$. Foram utilizadas as mesmas condições cromatográficas anteriores, embora com hélio como gás carreador (1,5 mL/min).

\section{Plantas analisadas}

As amostras analisadas constituem-se de folhas de andiroba (Carapa guianensis - Meliaceae), caapi (Banisteriopsis caapi Malpighiaceae), cacau (Theobroma cacao L. - Sterculiaceae), castanheira (Bertholletia excelsia - FAMILIA), cupuaçu (Theobroma grandiflorum - Sterculiaceae), marupá (Simaruba amara Simaroubaceae) e seringueira (Hevea brasiliensis - Euphorbiaceae). As folhas das referidas espécies foram coletadas e identificadas no Instituto Nacional de Pesquisa da Amazônia (INPA) em Manaus, pelos Drs. Cabral e Ferreira.

\section{Síntese dos padrões}

\section{Palmitato de fitila}

Em um balão de $5 \mathrm{~mL}$ equipado com um condensador de refluxo adicionou-se 0,3296 mmol $(100 \mu \mathrm{L})$ de cloreto de palmitoíla, $650 \mu \mathrm{L}$ de hexano, 0,4012 mmol (140 $\mu \mathrm{L})$ de fitol e $0,3204 \mathrm{mmol}$ $(45 \mu \mathrm{L})$ de trietilamina. Formou-se rapidamente um precipitado branco. A mistura reacional foi aquecida a $80{ }^{\circ} \mathrm{C}$ por $2 \mathrm{~h}$, depois foi resfriada e extraída com $2 \mathrm{~mL}$ de hexano (3 vezes). A solução hexânica foi transferida para outro frasco e o solvente evaporado sob fluxo de nitrogênio $\left(\mathrm{N}_{2}\right)$. Resultou um óleo esverdeado com odor característico. O óleo foi purificado através de CCD usando uma mistura de 9:1 de hexano e éter dietílico, como fase móvel e a fração de ésteres da placa $\left(\mathrm{R}_{\mathrm{f}} \sim 0,9\right)$ foi extraída com $\mathrm{CH}_{2} \mathrm{Cl}_{2}$ e o solvente evaporado com um fluxo de $\mathrm{N}_{2}$. Foi obtido um líquido incolor (que se solidificou em cristais brancos sob resfriamento). RMN de ${ }^{1} \mathrm{H}\left(\mathrm{CDCl}_{3}, 200 \mathrm{MHz}\right.$,

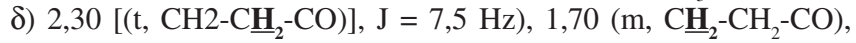
4,59 [(d, $\left.\left.-\underline{\mathrm{CH}}_{2}-\mathrm{O}-\mathrm{CO}, \mathrm{J}=7,1 \mathrm{~Hz}\right)\right], 5,34$ [(t, O-CH $-\mathrm{C} \underline{\mathbf{H}}=\mathrm{C}-, \mathrm{J}=7,1$ $\mathrm{Hz})], 2,01\left[\left(\mathrm{t},=\mathrm{C}\left(\mathrm{CH}_{3}\right)-\mathrm{CH}_{2}-\mathrm{CH}_{2}-, \mathrm{J}=7,5 \mathrm{~Hz}\right)\right] ; \mathrm{RMN} \mathrm{de}^{13} \mathrm{C}\left(\mathrm{CDCl}_{3}\right.$,

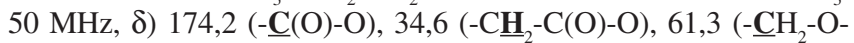
$\mathrm{CO}), 118,4\left(-\mathrm{CH}_{2}-\underline{\mathbf{C H}}=\mathrm{C}\left(\mathrm{CH}_{3}\right)-\right), 142,8\left(-\mathrm{CH}_{2}-\mathrm{CH}=\underline{\mathbf{C}}\left(\mathrm{CH}_{3}\right)-\right) . \mathrm{EM}$ $\mathrm{m} / \mathrm{z},(\%): \mathrm{M}^{+} 534$ (1,1), 123 (100), 71 (57), 95 (71), 278 (16), 296 (5); EMAR 534,5378 Da, calculado para $\left(\mathrm{C}_{36} \mathrm{H}_{70} \mathrm{O}_{2}\right)$ 534,5375 Da.

\section{Palmitato de $\alpha$-tocoferila}

Em um balão de $5 \mathrm{~mL}$ equipado com um condensador de refluxo foi adicionado 0,2554 mmol (110,1 mg) de $\alpha$-tocoferol, $250 \mu \mathrm{L}$ de hexano e $0,2802 \mathrm{mmol}(85 \mu \mathrm{L})$ de cloreto de palmitó́la. A mistura foi aquecida a $80{ }^{\circ} \mathrm{C}$ por $2 \mathrm{~h}$. Ao final do aquecimento formaram-se cristais brancos no balão. Os cristais foram purificados através de CCD usando uma mistura de 9:1 de hexano e éter dietílico e a fração de ésteres $\left(\mathrm{R}_{\mathrm{f}} \sim 0,9\right)$ extraída com $\mathrm{CH}_{2} \mathrm{Cl}_{2}$ e o solvente foi evaporado sob fluxo de $\mathrm{N}_{2}$. RMN de ${ }^{1} \mathrm{H}\left(\mathrm{CDCl}_{3}, 200 \mathrm{MHz}, \delta\right) 0,88$ [(d, $\mathrm{R}_{1}-$ $\left.\mathrm{CH}\left(\mathrm{CH}_{3}\right)-\mathrm{R}_{2}, \mathrm{~J}=6,4 \mathrm{~Hz}\right)$ ], 2,60 [(t, R-CH $\left.-\mathrm{CH}_{2}-\mathrm{C}(\mathrm{O})-\mathrm{O}, 7,3 \mathrm{~Hz}\right)$ ], 1,77 [(m, R-C $\left.\left.\underline{\mathbf{H}}_{2}-\mathrm{CH}_{2}-\mathrm{C}(\mathrm{O})-\mathrm{O}\right)\right], 1,97\left[\left(\mathrm{~s}, \mathrm{CH}_{3}\right.\right.$ aromático $\left.)\right], 2,02$ [(s, $\mathrm{CH}_{3}$ aromático)], 2,09 [(s, $\mathrm{CH}_{3}$ aromático)]; $\mathrm{RMN}$ de ${ }^{13} \mathrm{C}\left(\mathrm{CDCl}_{3}\right.$, $50 \mathrm{MHz}, \delta)$ 172,6 (R-CH $-\underline{\mathrm{C}}(\mathrm{O})-\mathrm{O}-), 34,4$ (R- $\left.\underline{\mathrm{CH}}_{2}-\mathrm{C}(\mathrm{O})-\mathrm{O}-\right), 149,5$, 140,6, 126,8, 125,1, 123,2, 117,5 (C-aromáticos). EM m/z (\%): M+• 668 (0,9), 416 (100), 165 (36), 57 (8), 205 (3), EMAR 668,6104 Da, calculado para $\left(\mathrm{C}_{45} \mathrm{H}_{80} \mathrm{O}_{3}\right) 668,6107 \mathrm{Da}$.

\section{RESULTADOS E DISCUSSÃO}

A análise dos extratos brutos de diclorometano e as frações resultantes de CCD das diversas espécies investigadas revelaram uma grande diversidade de classes de compostos de massa molecular alta. Estes compostos estão presentes em todas as plantas analisadas, contudo diferem na quantidade, na funcionalidade química, na estrutura e na variedade de homólogos presentes.

\section{Ésteres graxos saturados}

Os ésteres graxos (ésteres de ácidos e álcoois de cadeias longas) formam uma das principais classes na composição das ceras epicuticulares. Essa classe é composta, principalmente, por derivados do ácido palmítico $\left(\mathrm{C}_{16}\right)$. Os ésteres graxos são caracterizados pela espectrometria de massas através dos seus picos bases, que são gerados a partir de um rearranjo com transferência dupla de hidrogênio, formando um íon de razão $\mathrm{m} / \mathrm{z}$ com massa molecular do ácido mais um hidrogênio $\left[\mathrm{M}_{\mathrm{Ac}}\right.$ (massa molecular do ácido $\left.)+1\right]^{7}$. Assim, pode-se determinar a presença de ésteres graxos dos ácidos hexadecanóico $\left(\mathrm{C}_{16}\right)$, octadecanóico $\left(\mathrm{C}_{18}\right)$, eicosanóico $\left(\mathrm{C}_{20}\right)$, respectivamente, a partir dos íons de $m / z, 257,285$ e 313, e assim também para outros ésteres graxos de ácidos lineares passíveis de rearranjos.

Todas as amostras analisadas apresentam séries homólogas pares de carbono de ésteres graxos do ácido hexadecanóico, sendo que a série mais extensa é observada na amostra marupá. Esta amostra possui uma série de hexadecanoatos (palmitatos) que se inicia com a esterificação do álcool tetradecanol $\left(\mathrm{C}_{14}\right)$ e se estende até ao álcool tetratriacontanol $\left(\mathrm{C}_{34}\right)$. As demais amostras apresentam séries mais curtas.

A Tabela 1 apresenta a relação de todas as séries de hexadecanoatos caracterizadas nas amostras analisadas. Nela podemos observar que são formados íons característicos de alcanos lineares $\mathrm{m} / \mathrm{z}$ 57 e 71 , devido à linearidade dos compostos. Também observamos a formação, em menor intensidade, dos íons de $\mathrm{m} / z 239$ e $\mathrm{m} / \mathrm{z}$ 258. É sabido a formação dos cetenos em ésteres graxos, nesse caso correspondendo ao íon de $m / z, 258$, mas os dois íons apresentam-se em todos os espectros, diferenciando apenas nas abundâncias relativas. Um fragmentograma de $\mathrm{m} / z, 257$ representativo para toda a série é apresentado na Figura 1.

Além dos ésteres graxos do ácido hexadecanóico $\left(\mathrm{C}_{16}\right)$, também estão presentes ésteres de outros ácidos, como o octadecanóico $\left(\mathrm{C}_{18}\right)$, eicosanóico $\left(\mathrm{C}_{20}\right)$, dodeicosanóico $\left(\mathrm{C}_{22}\right)$ e o tetraeicosanóico $\left(\mathrm{C}_{24}\right)$. Assim como os ésteres do ácido hexadecanóico, os demais ésteres graxos são caracterizados, nos espectros de massas, pelo pico base que é o íon formado pelo fragmento relativo à massa do ácido mais uma unidade de massa, ou seja, um íon de $m / z,\left[\mathbf{M}_{\mathrm{Ac}}+1\right]$. Dessa forma, os íons característicos para ésteres dos ácidos octadecanóico, eicosanóico, dodeicosanóico e tetraeicosanóico são, respectivamente, $\mathrm{m} / \mathrm{z}, 285,313,341,369$. Com base nesses íons foi feito um estudo de fragmentografia nas amostras analisadas onde foi observada a presen-

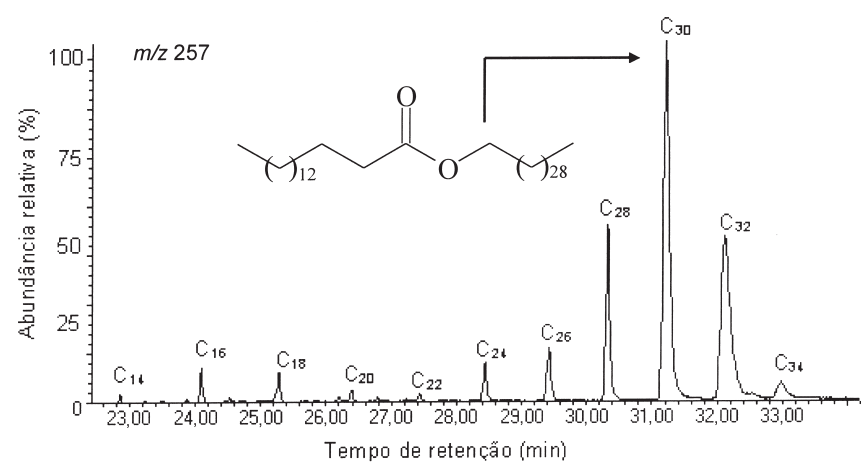

Figura 1. Fragmentograma m/z 257 da amostra marupá, característico dos hexadecanoatos e representativo para todas as amostras. Os números representam o número de carbonos da cadeia dos álcoois esterificados 
Tabela 1. Relação dos ésteres do ácido hexadecanóico (palmitatos), encontrados em plantas da Amazônia, representativos dos ésteres de ácidos graxos com álcoois de cadeia longa. Álcoois variam de $\mathrm{C}_{14}$ até $\mathrm{C}_{34}$

\begin{tabular}{|c|c|c|c|c|c|c|c|c|}
\hline $\begin{array}{c}\mathrm{t}_{\mathrm{R}}(\min ) \\
\operatorname{mar}\end{array}$ & $\begin{array}{c}\mathrm{t}_{\mathrm{R}}(\min ) \\
\text { cast }\end{array}$ & $\begin{array}{l}\mathrm{t}_{\mathrm{R}}(\min ) \\
\text { and }\end{array}$ & $\begin{array}{c}\mathrm{t}_{\mathrm{R}}(\min ) \\
\operatorname{cup}\end{array}$ & $\begin{array}{c}\mathrm{t}_{\mathrm{R}}(\min ) \\
\text { cac }\end{array}$ & $\begin{array}{c}\mathrm{t}_{\mathrm{R}}(\min ) \\
\text { ser }\end{array}$ & $\begin{array}{c}\mathrm{t}_{\mathrm{R}}(\min ) \\
\text { cap }\end{array}$ & Compostos & Íons Principais \\
\hline 22,9 & nd & nd & nd & nd & nd & nd & Palmitato de tetradecila & $452(257,239,71,57)$ \\
\hline 24,1 & nd & nd & nd & nd & nd & nd & Palmitato de hexadecila & $480(257,239,71,57)$ \\
\hline 25,2 & 25,8 & nd & nd & nd & nd & nd & Palmitato de octadecila & $508(257,239,71,57)$ \\
\hline 26,3 & 27,2 & nd & nd & nd & nd & nd & Palmitato de eicosila & $536(257,239,71,57$ \\
\hline 27,4 & 28,2 & nd & nd & nd & nd & 29,6 & Palmitato de dodeicosila & $564(257,258,71,57)$ \\
\hline 28,4 & 29,0 & 30,4 & 30,5 & 29,8 & nd & 30,6 & Palmitato de tetracosila & $592(257,258,71,57)$ \\
\hline 29,4 & 23,0 & 31,4 & 31,5 & 31,5 & 31,5 & 31,6 & Palmitato de hexacosila & $620(257,258,71,57)$ \\
\hline 30,2 & 30,9 & 32,4 & 32,4 & 32,4 & 32,4 & 32,5 & Palmitato de octacosila & $648(257,258,71,57)$ \\
\hline 31,2 & 31,8 & 33,3 & 33,3 & 33,3 & 33,3 & 33,4 & Palmitato de triacontila & $676(257,258,71,57)$ \\
\hline 32,1 & 32,7 & 34,1 & 34,2 & 34,1 & 34,1 & 34,3 & Palmitato dodetriacontila & $704 *(257,258,71,57)$ \\
\hline 33,0 & nd & nd & 35,0 & 35,0 & 35,0 & nd & Palmitato tetratriacontila & $732 *(257,258,71,57)$ \\
\hline
\end{tabular}

*Valores obtidos por extrapolação da série. mar = marupá; cast = castanheira; and = andiroba; cup = cupuaçu; cac = cacau; ser = seringueira; cap = caapi; nd = não detectado.

ça desses fragmentos em um mesmo espectro de massas, evidenciando a co-eluição dos ésteres graxos, de ácidos e álcoois diferentes, mas com mesma massa molecular. Reiter e colaboradores ${ }^{7}$ verificaram esse fato na análise de óleos vegetais, onde as diversas possibilidades de combinações de ácidos e álcoois, formando ésteres com mesma massa molecular, forneciam espectros de massas com diversos fragmentos característicos. A Figura 2 apresenta fragmentogramas (2b) e um espectro de massas (2d) evidenciando a aparente co-eluição dos ésteres graxos com mesma massa molecular, e inclusive, a presença de outras séries minoritárias ( $\mathrm{m} / \mathrm{z}, 397$ etc.). Assim, essa aparente co-eluição dos picos isobáricos resulta do "envelope" formado pela eluição próxima de todos os isômeros, evidenciada pela não co-eluição dos máximos dos picos dos fragmentogramas (Figura 2c).

\section{Ésteres terpênicos}

Os terpenóides constituem uma das mais diversificadas classes de compostos químicos caracterizadas em extratos vegetais. Apresentam a mesma origem biossintética a partir do ácido mevalônico e, mais recentemente, foi proposta a rota DOXF-desoxi-D-Xilulose-5fosfato $\left[\mathrm{DOXP}(1-\text { deoxy-D-xylulose-5-phosphate) }]^{8}\right.$. São responsáveis por uma grande diversidade de esqueletos hidrocarbônicos (es-
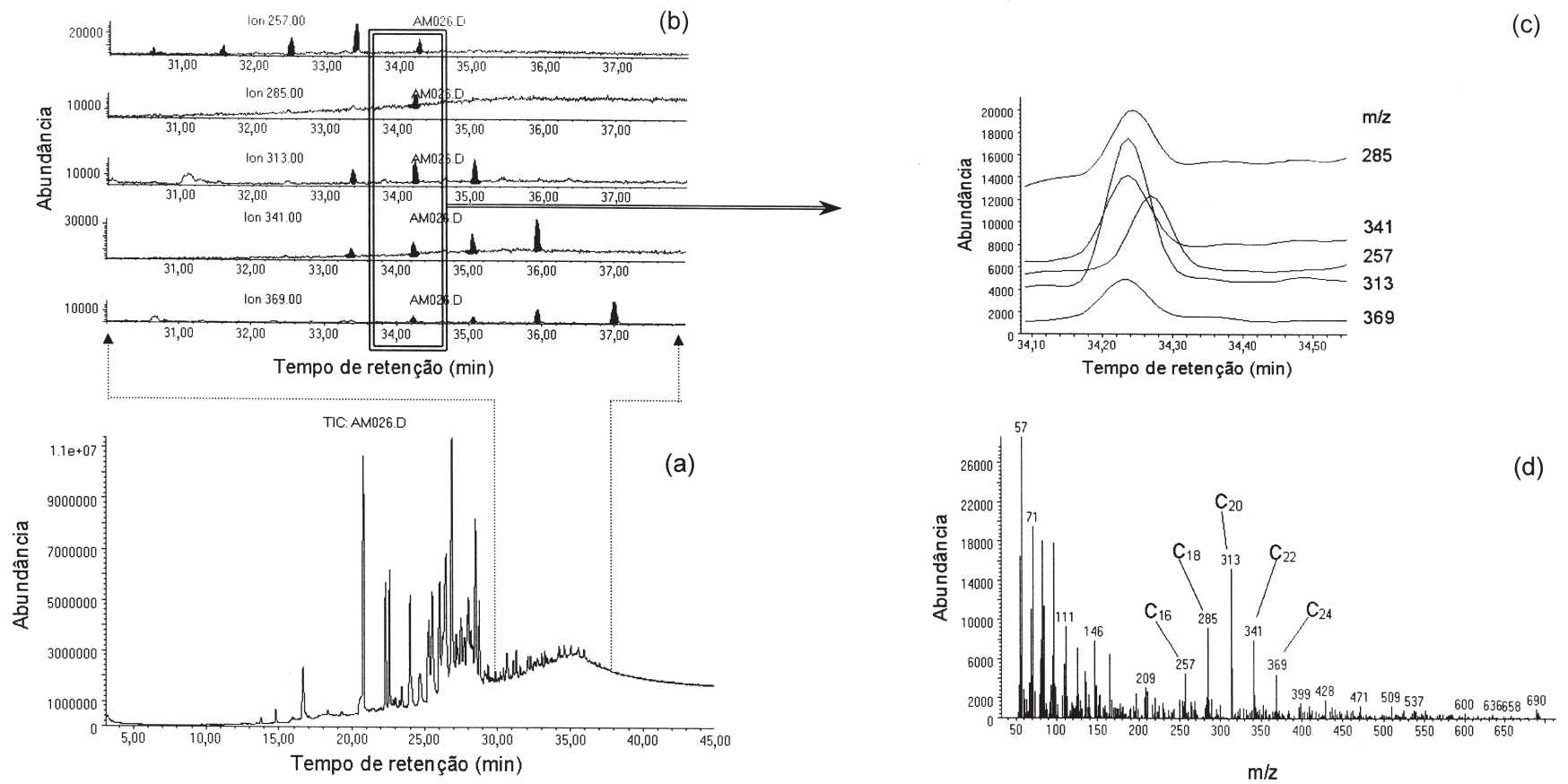

Figura 2. (a) Cromatograma de íons totais (CIT) da amostra caapi; (b) Fragmentogramas de razão m/z 257, 285, 313, 341, 369 característicos dos ésteres dos ácidos hexadecanóico $\left(C_{16}\right)$, octadecanóico $\left(C_{18}\right)$, eicosanóico $\left(C_{20}\right)$, dodeicosanóico $\left(C_{22}\right)$ e tetraeicosanóico $\left(C_{24}\right)$, evidenciando a co-eluição dos ésteres graxos na amostra caapi. Os ésteres dos diferentes ácidos com o mesmo valor de $t_{R}$ são compostos isobáricos, pois são esterificados por álcoois com cadeias hidrocarbônicas complementares, formando, ao final, ésteres com mesma massa molecular; (c) expansão do pico localizado em 34,25 min, ilustra a não co-eluição dos máximos dos ésteres isobáricos, gerando o alargamento do pico observado no cromatograma de íons totais (detalhe); (d) espectro de massas, obtido no $t_{R}=34,25 \mathrm{~min}$, apresentando os fragmentos de razão $\mathrm{m} / \mathrm{z} 257,285,313,341$ e 369. Os índices correspondem ao comprimento da cadeia da parte ácida do éster, a qual complementada pelos álcoois, resulta em $\mathrm{M}^{+\bullet}$ de $\mathrm{m} / \mathrm{z} 705\left(\mathrm{C}_{48} \mathrm{H}_{96} \mathrm{O}_{2}\right)$ 
truturas), proporcionando um enorme potencial para as interações fisiológicas e ecológicas das plantas. Embora todas as plantas sintetizem terpenóides, necessários para seu crescimento e desenvolvimento, alguns desses compostos são específicos e característicos de algumas espécies ou mesmo famílias de plantas, de meio ambiente específico. Essa grande variedade de estruturas também possibilita diferentes ações, tais como, defesa contra insetos, animais herbívoros e fungos patogênicos, atração de agentes polinizadores e interações com os gases atmosféricos entre outras?

Nos extratos analisados foram caracterizados vários compostos triterpênicos. Os picos mais intensos nos cromatogramas de íons totais (CIT), que geraram espectros de massas com características triterpênicas, na espécie castanheira, correspondem a duas séries de triterpenos pentacíclicos homólogos, as $\alpha$ e $\beta$-amirinas. Elias e colaboradores ${ }^{3,10}$ determinaram esses mesmos triterpenos esterificados em amostras de aerossóis em queimada controlada. Elias e colaboradores propõem a utilização desses compostos como marcadores diferenciais nas queimadas de matéria orgânica biogênica e antropogênica, contudo ressaltavam a necessidade de verificar a presença dos ésteres triterpênicos na matriz vegetal, para certificar de que não se constituía de um artefato produzido durante a combustão.

O presente trabalho permitiu observar, nas plantas estudadas, que os ésteres apresentam uma forte predominância de homólogos da série par sobre a ímpar, com o destaque para as amirinas esterificadas com o ácido palmítico $\left(\mathrm{C}_{16}\right)$. A interpretação dos espectros de massas desses compostos é simples. Essencialmente, consiste na formação do íon molecular $\left(\mathrm{M}^{+*}\right)$, uma perda de metila $(\mathrm{M}$ $\mathrm{CH}_{3}$ ), a perda do ácido graxo (M - ác. graxo) e a fragmentação característica dos triterpenos pentacíclicos. Elias e colaboradores ${ }^{2}$ propõem uma rota de fragmentação para estes compostos. A Figura 3 apresenta o fragmentograma e o espectro de massas do palmitato de $\beta$-amirina como representante dos compostos da série.

(c)
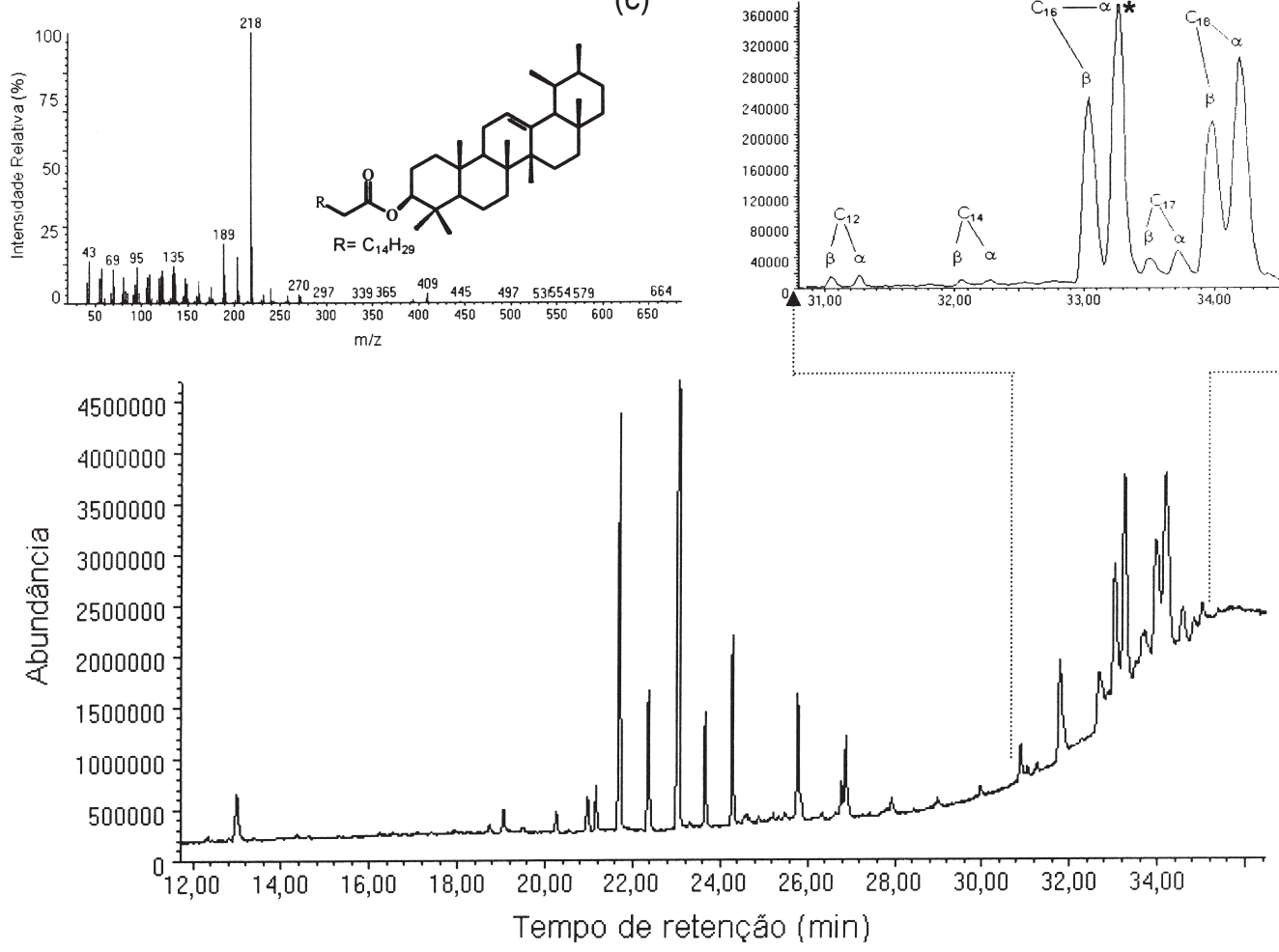

Outro terpenóide muito freqüente em extratos de plantas é o fitol. Este diterpeno está presente em todos vegetais que apresentam clorofila, pois é um constituinte na molécula de clorofila. A análise dos extratos por CGAR-AT-EM das espécies andiroba, caapi, cacau, cupuaçu e marupá revelou a presença de uma série de compostos que apresentavam em comum o fragmento $\mathrm{m} / \mathrm{z}, 123$ entre outros. A determinação dessa série como sendo de ésteres do fitol foi feita mediante o emprego de um padrão sintético, o palmitato de fitila.

O espectro de massas do produto sintetizado apresenta como íons principais, em abundância e importância para a elucidação estrutural, os íons de razão $m / z$, 95, 123, 278, 296 que são fragmentos característicos da parte fitílica do éster, o $\mathrm{m} / \mathrm{z} 239$ e o 256 da parte ácida e o $\mathrm{M}^{+\bullet} 534$, conforme o espectro da Figura 4. O Esquema 1 apresenta uma proposta de fragmentação padrão para esse composto e para toda a série que ele representa, procurando interpretar os íons observados no espectro. Duas rotas de fragmentação são propostas nesse esquema. Na primeira rota sugere-se a formação de um grupo ceteno, a partir do ácido esterificante, através de uma reação de transferência de hidrogênio do carbono $\alpha$ à carbonila do éster para o oxigênio do fitol, formando o íon fitol $(\mathrm{m} / \mathrm{z}, 296)$. O íon fitol perde uma molécula de $\mathrm{H}_{2} \mathrm{O}$ formando por sua vez o íon $\mathrm{m} / z$.278, que sofre sucessivas fragmentações formando os íons de $m / z .123$ e 71 . A segunda rota de fragmentação tem a clivagem da ligação éster por um mecanismo de transferência de $\mathrm{H}$ (hidrogênio), via rearranjo de McLafferty, formando uma molécula do ácido palmítico e o íon $\mathrm{m} / z, 278$ e conseqüente formação dos íons $\mathrm{m} / z$, 123 e 95 . Os íons apresentados nesta proposta de fragmentação também são observados quando o palmitato de fitila é analisado por EM-AR. A massa do íon molecular observada no espectro de massas de alta resolução é de 534,5378 Da e a massa calculada para o compostos com fórmula molecular $\mathrm{C}_{36} \mathrm{H}_{70} \mathrm{O}_{2}$ (hexadecanoato de fitila) é de 534,5375 Da, obtendo-se assim, uma diferença entre a massa observada e a calculada de $0,3 \mathrm{mDa}$, o que

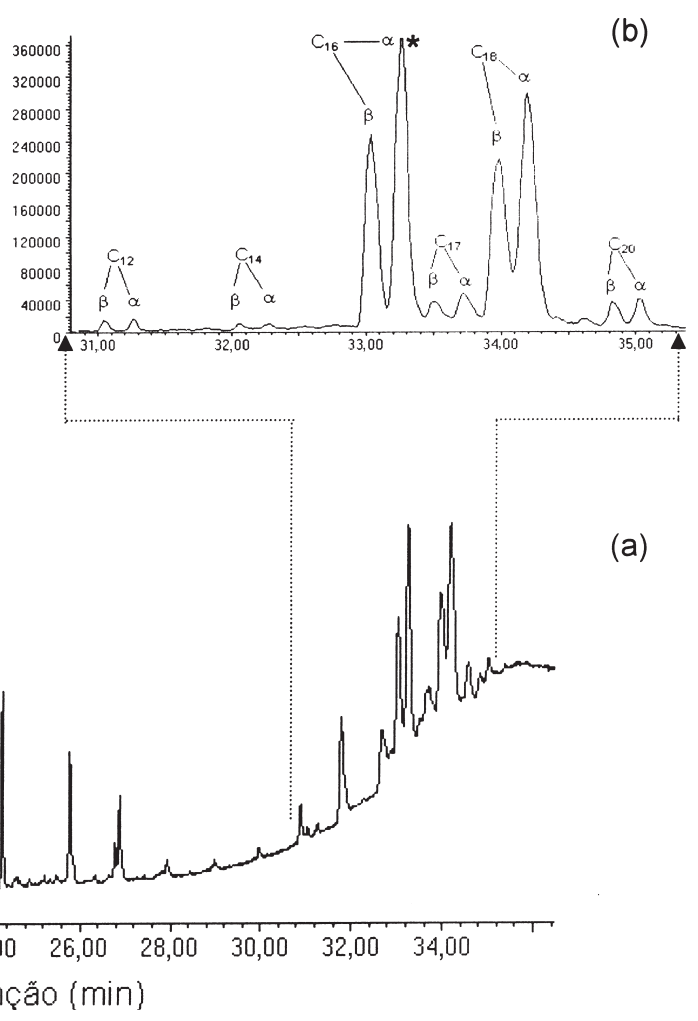

Figura 3. (a) Cromatograma de íons totais da fração de ésteres da amostra castanheira; (b) fragmentograma $\mathrm{m} / \mathrm{z} 218$ característico dos ésteres das $\alpha$ e $\beta$ amirinas. Os índices indicam o tamanho da cadeia dos ácidos esterificantes e $\alpha$ e $\beta$ são os triterpenóides $\alpha$ e $\beta$-amirina, respectivamente; (c) espectro de massas do palmitato de $\alpha$-amirina* da amostra castanheira $\left(t_{R}=33,3 \mathrm{~min}\right)$ 

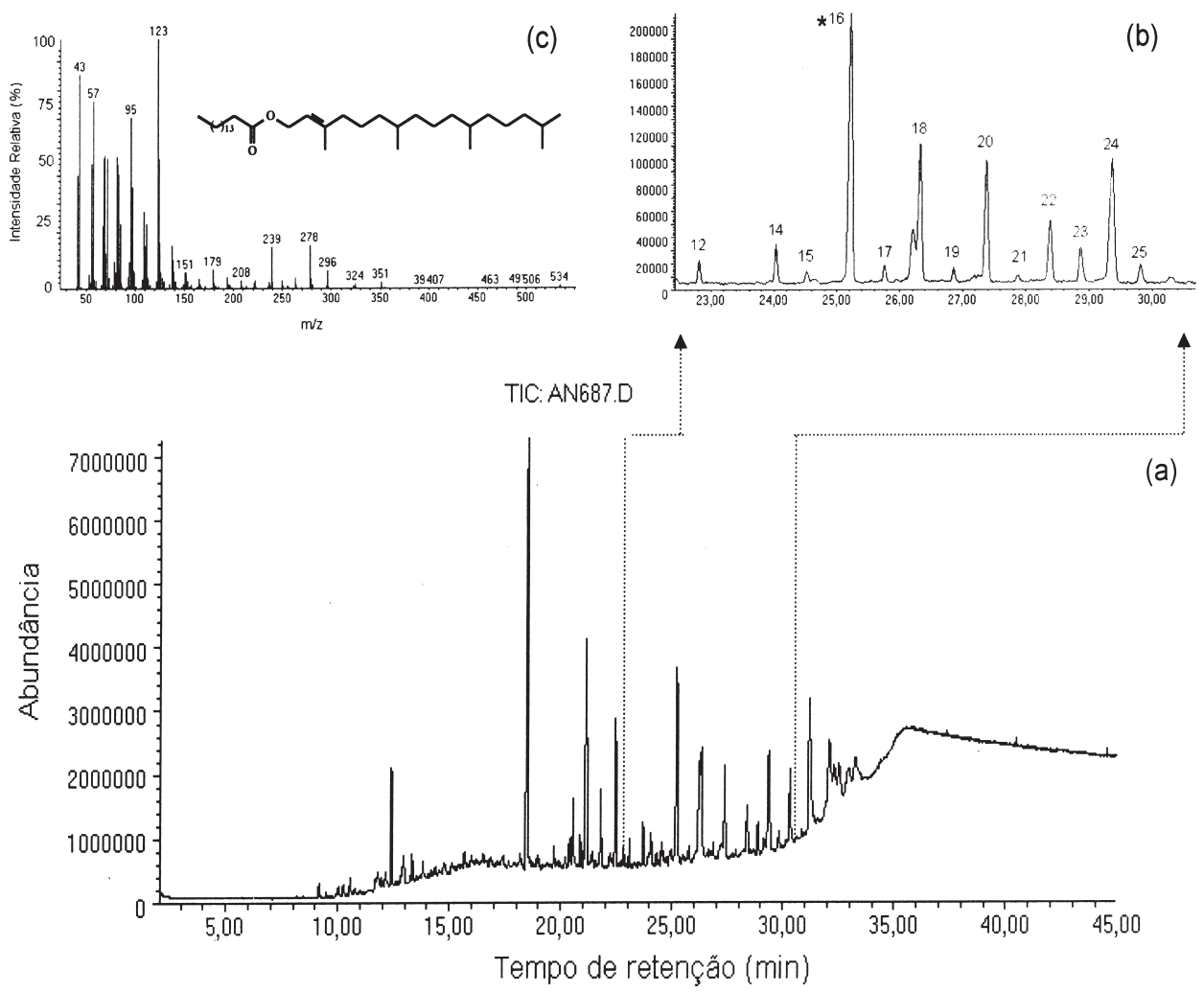

Figura 4. (a) Cromatograma de íons totais (CIT) da amostra marupá (fração de ésteres); (b) fragmentograma de m/z 123 característico dos ésteres do fitol, os números indicam o comprimento da cadeia do ácido graxo esterificante; (c) espectro de massas do palmitato de fitila $*$ da amostra marupá ( $t_{R}=25,2$ min)

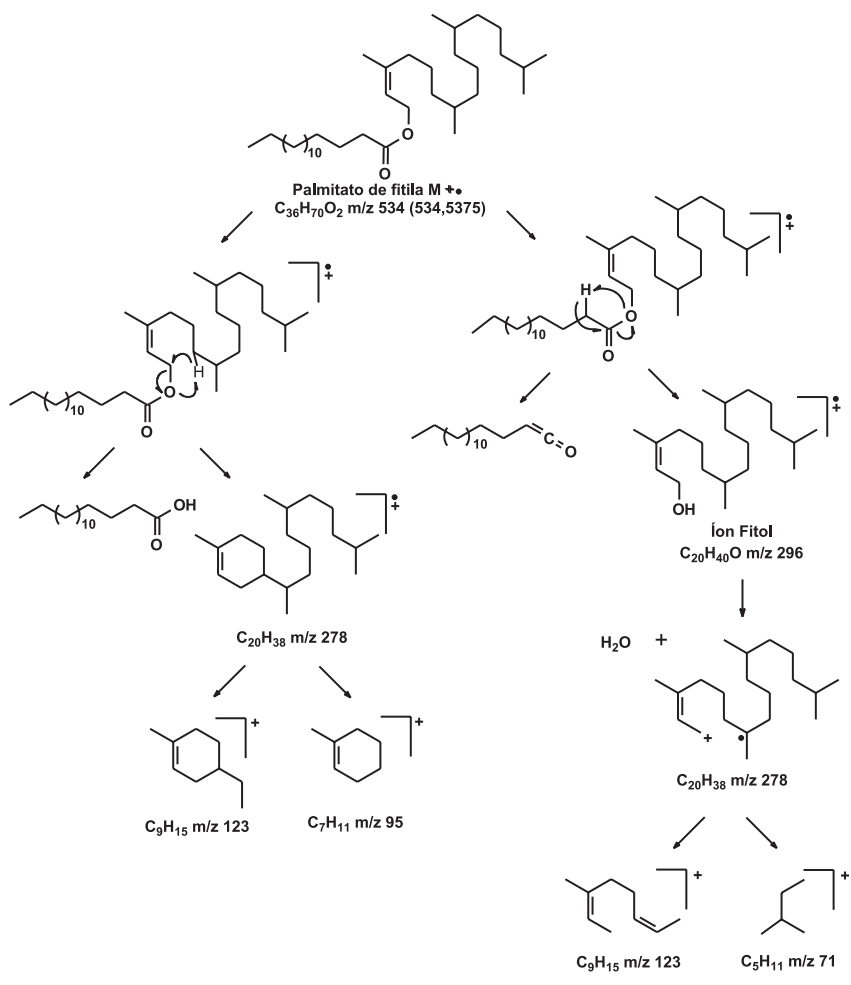

Esquema 1. Rota de fragmentação padrão proposta para os ésteres do fitol. Adapatada da ref. 19. Pequenas impropriedades da versão original foram corrigidas caracteriza como sendo o composto analisado o hexadecanoato de fitila. Os dados de RMN de ${ }^{13} \mathrm{C}$ e ${ }^{1} \mathrm{H}$ e IV do palmitato de fitila natural coincidem com os dados observados para o produto sintético.

A série de ácidos graxos que esterificam o fitol apresenta predominância dos homólogos pares sobre os ímpares, apresentando frequiência maior no palmitato de fitila. A ocorrência do palmitato de fitila havia sido descrita previamente em extratos de folhas de Pentatropis spirallis por Rasool e colaboradores ${ }^{11}$, Leucas nutans por Mashooda e colaboradore ${ }^{12}$ e, mais recentemente, a determinação de uma série de homólogos pares com cinco membros, a partir do ácido $\mathrm{C}_{16}$ (hexadecanóico) até o $\mathrm{C}_{24}$ (tetraeicosanóico), em extratos de Vicia faba por Griffiths e colaboradores ${ }^{13}$. Contudo, a série apresentada neste trabalho é mais complexa do que a determinada anteriormente, sendo mais extensa e incluindo a presença de homólogos ímpares. No presente caso, a identificação é inequívoca, pois envolve padrão identificado e a utilização de dados da EM-AR. A Tabela 2 apresenta a relação dos compostos identificados.

\section{Ésteres do tocoferol}

A proteção das plantas contra danos causados pela radiação ultravioleta, radicais livres e agentes químicos é atribuída em parte pela presença de substâncias anti-oxidantes como os carotenóides e as vitaminas $\mathrm{C}$ e $\mathrm{E}$ (tocoferol). Essa proteção fornecida devida ao tocoferol e carotenóides tem sido, também, observada em tecido epidérmico humano. Importantes resultados têm sido obtidos com os tocoferóis na prevenção de doenças cardiovasculares e no tratamento de cataratas oftalmológicas ${ }^{14-17}$. As formas mais comuns de tocoferóis, encontradas na natureza e já estudadas, são o álcool livre e a acetilada, possivelmente devido à metodologia analítica empre- 
Tabela 2. Relação dos ésteres do fitol encontrados em plantas da Amazônia. Os ácidos graxos variam de $\mathrm{C}_{12}$ até $\mathrm{C}_{14}$

\begin{tabular}{cccccll}
\hline $\begin{array}{c}\mathrm{t}_{\mathrm{R}}(\min ) \\
\text { and }\end{array}$ & $\begin{array}{c}\mathrm{t}_{\mathrm{R}}(\min ) \\
\text { cup }\end{array}$ & $\begin{array}{c}\mathrm{t}_{\mathrm{R}}(\mathrm{min}) \\
\mathrm{mar}\end{array}$ & $\begin{array}{c}\mathrm{t}_{\mathrm{R}}(\mathrm{min}) \\
\text { cac }\end{array}$ & $\begin{array}{c}\mathrm{t}_{\mathrm{R}}(\mathrm{min}) \\
\text { cap }\end{array}$ & Compostos caracterizados & $\begin{array}{l}\text { Íons principais } \\
m / z\end{array}$ \\
\hline 18,8 & 16,5 & 18,5 & 18,8 & 18,8 & Fitol & $296(71,95,123,278)$ \\
nd & nd & 22,8 & nd & 26,6 & Dodecanoato de fitila & $478(57,71,95,123,278,296)$ \\
nd & nd & nd & nd & 27,1 & Tridecanoato de fitila & $492(57,71,95,123,278,296)$ \\
nd & nd & 24,0 & nd & 27,8 & Tetradecanoato de fitila & $506(57,71,95,123,278,296)$ \\
nd & nd & 24,6 & nd & 28,4 & Pentadecanoato de fitila & $520(57,71,95,123,278,296)$ \\
27,0 & 27,0 & 25,2 & 29,4 & 29,1 & Hexadecanoato de fitila & $534(57,71,95,123,278,296)$ \\
nd & 27,6 & 25,8 & nd & 29,6 & Heptadecanoato de fitila & $548(57,71,95,123278,296)$ \\
28,1 & 28,2 & 26,3 & 30,5 & 30,2 & Octadecanoato de fitila & $562(57,71,95,123,278,296)$ \\
nd & 28,7 & 26,8 & nd & nd & Nonadecanoato de fitila & $576(57,71,95,123,263,278)$ \\
nd & 29,3 & 27,4 & 31,6 & 31,3 & Eicosanoato de fitila & $590(57,71,95,123,278,296)$ \\
nd & 29,8 & 27,9 & nd & nd & Heneicosanoato de fitila & $604(57,71,95,123,278,296)$ \\
nd & 30,3 & 28,4 & 32,5 & 32,3 & Dodeicosanoato de fitila & $618(57,71,95,123,278,296)$ \\
nd & 30,8 & 28,8 & nd & nd & Tricosanoato de fitila & $632(57,71,95,123,278,296)$ \\
nd & 31,3 & 29,4 & 33,5 & nd & Tetracosanoato de fitila & $646(57,71,95,123,278,296)$ \\
nd & nd & 29,8 & nd & nd & Pentacosanoato de fitila & $660(57,71,95,123,278,296)$ \\
\hline
\end{tabular}

*Valores obtidos por extrapolação da série; and = andiroba; cup = cupuaçu; mar = marupá; cac = cacau; cap = caapi; nd = não detectado.

gada. Utilizando o sistema a CGAR-AT-EM, uma série de ésteres graxos do alfa $(\alpha)$ tocoferol foi determinada em extratos da andiroba, caapi, cacau, cupuaçu, marupá e seringueira. No extrato de caapi foi observada a ocorrência adicional de ésteres graxos dos tocoferóis beta, gama e delta.

$\mathrm{O}$ estudo da fragmentografia nos extratos analisados revelou a presença de uma série de compostos que apresentava os íons de $\mathrm{m} / \mathrm{z}$. 165 e 430 . Estes fragmentos são característicos do $\alpha$-tocoferol. Além desses dois fragmentos característicos, os espectros de massas apresentavam íons moleculares condizentes com uma variação linear de massa de uma possível esterificação do tocoferol por ácidos graxos. Para realizar uma identificação inequívoca da série, foi preparado um padrão sintético do palmitato do $\alpha$-tocoferol.

$\mathrm{O}$ espectro de massas do produto de síntese apresenta como fragmentos principais íons de $\mathrm{m} / \mathrm{z}$ os íons 165, 430 e 668. Poter e Baldas ${ }^{18}$ propõem uma rota de fragmentação para o acetato de tocoferila, a qual pode ser estendida à fragmentação dos ésteres graxos. A partir do íon molecular 668 do palmitato de tocoferol, tem-se a formação do fragmento de $m / z 430$ através da perda de um ceteno do ácido esterificante. A formação do ceteno ocorre através de um rearranjo de hidrogênio com a transferência do mesmo, do carbono $\alpha$ à carbonila para o oxigênio do tocoferol. O íon de $\mathrm{m} / \mathrm{z} 430$ formado sofre um processo de fragmentação através de um rearranjo de hidrogênio (rH) uma reação de retro Diels-Alder (RDA) no anel pirânico do tocoferol, para dar origem ao íon $\mathrm{m} / \mathrm{z}, 165$, ou a perda da cadeia alquílica lateral para formar o fragmento $\mathrm{m} / \mathrm{z} 205$. Outra alternativa na formação do íon $\mathrm{m} / \mathrm{z}, 165$ é a fragmentação a partir do íon molecular por rearranjo de hidrogênio $(\mathrm{rH})$ e uma reação de retro Diels-Alder (RDA) para formar o íon $\mathrm{m} / \mathrm{z} 403$ de baixa intensidade, e posterior perda do ceteno para dar origem ao íon $\mathrm{m} / \mathrm{z}$ 165. A rota de fragmentação proposta para a formação dos íons, apresentada no Esquema 2, é evidenciada pelos dados obtidos no espectro de EMAR. O íon molecular apresenta uma massa calculada de 668,6107 Da, enquanto que a massa observada no espectro tem $\mathrm{m} / z$, 668,6104 Da $\left(\mathrm{C}_{45} \mathrm{H}_{80} \mathrm{O}_{3}\right)$ apresentando uma diferença de 0,3 mDA. Com base nesta diferença de massa, pode-se afirmar que a fórmula molecular do composto analisado é $\mathrm{C}_{45} \mathrm{H}_{80} \mathrm{O}_{3}$. Os dados de $\mathrm{RMN}{ }^{1} \mathrm{H} \mathrm{e}^{13} \mathrm{C}$ e IV-TF corroboram na identificação da série desses ésteres, pela comparação com o palmitato de tocoferila sintético.

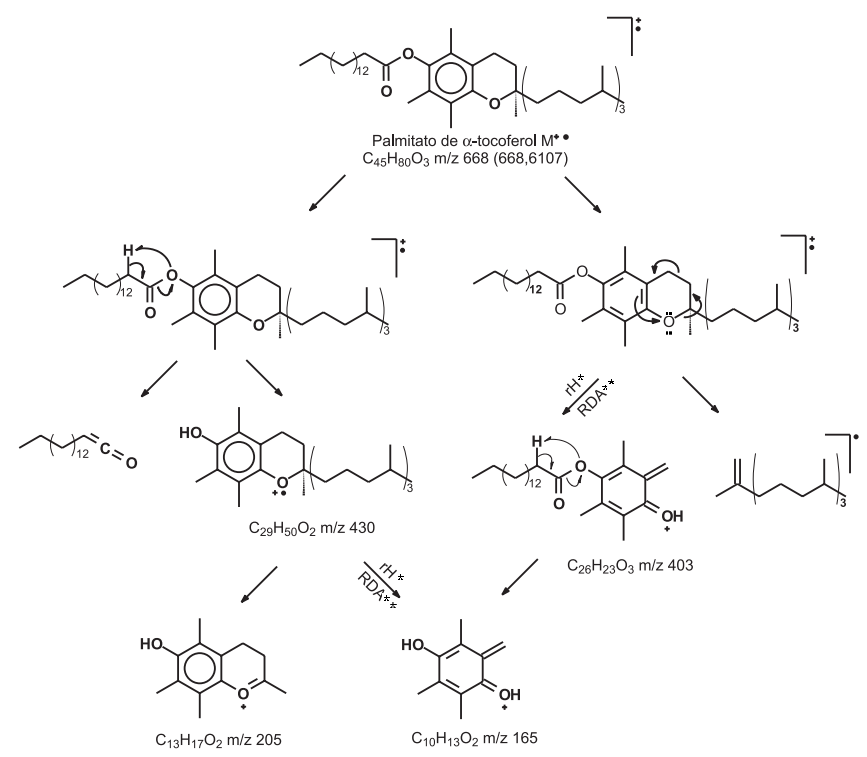

Esquema 2. Rota de fragmentação padrão proposta para os ésteres do $\alpha$ tocoferol. Adaptado da ref. 18. * rearranjo de hidrogênio; **retro DielsAlder

Para os demais tocoferóis foram feitos os mesmos estudos de fragmentografia, com exceção da síntese dos padrões. Naturalmente, devemos observar mudanças de alguns íons nos estudos da espectrometria de massas dos ésteres dos demais tocoferóis, face às diferenças de massas moleculares entre os compostos decorrentes das alterações estruturais que sofrem. Os tocoferóis beta e gama apresentam fragmentos característicos de $\mathrm{m} / \mathrm{z} 151$ e 416 (Figura 5) e o delta tocoferol apresenta fragmentos característicos, nos espectros de massas, de $m / z, 137$ e 402 (Figura 6). A Tabela 3 apresenta a relação dos ésteres do $\alpha$-tocoferol identificados. 

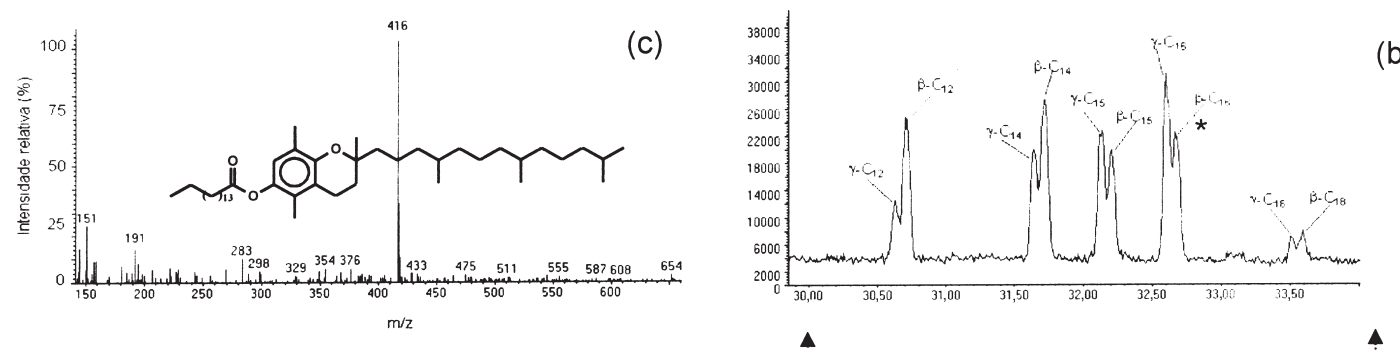

(b)

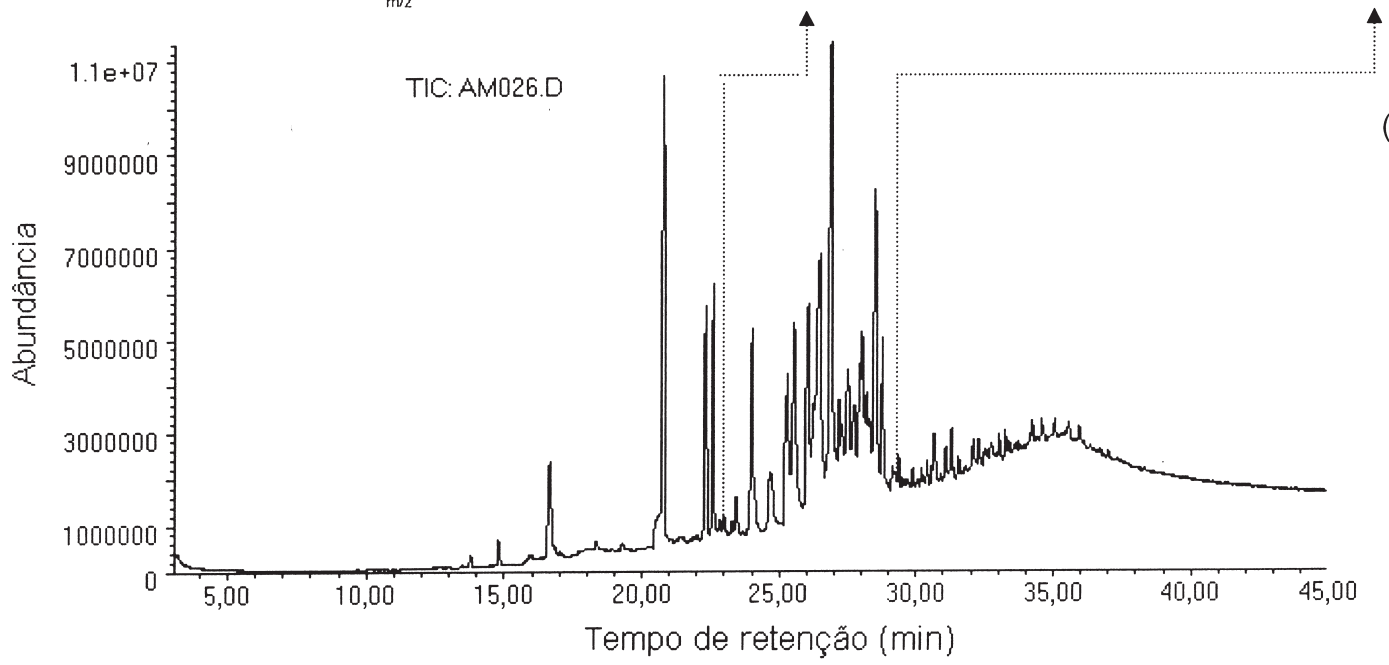

Figura 5. (a) Cromatograma de íons totais (CIT) da amostra caapi; (b) fragmentograma $\mathrm{m} / \mathrm{z} 416$ característico dos ésteres do $\beta$ e $\gamma$-tocoferóis, do extrato da amostra caapi. Os índices indicam o tamanho da cadeia do ácido esterificante do tocoferol; (c) espectro de massas do palmitato de $\beta$-tocoferol* $\left(t_{R}=32,7\right.$ min)

(c)
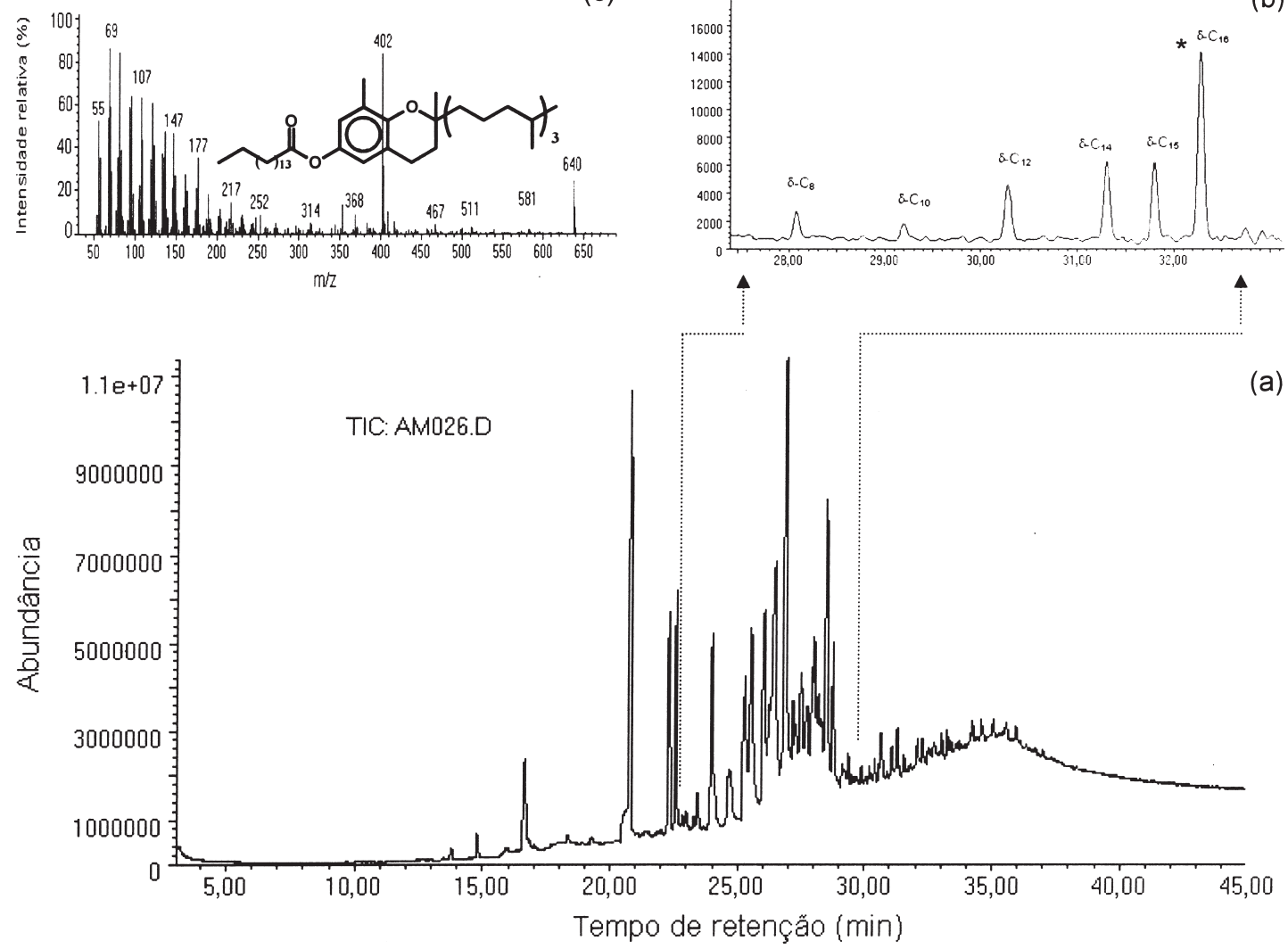

Figura 6. (a) Cromatograma de íons totais (CIT) da amostra caapi; (b) fragmentograma $\mathrm{m} / \mathrm{z} 402$ característico dos ésteres do $\delta$-tocoferol, do extrato da amostra caapi. Os índices indicam o tamanho da cadeia do ácido esterificante do tocoferol; $\left(\right.$ c) espectro de massas do palmitato de $\delta$-tocoferol $*$ ( $t_{R}=32,3$ $\min )$ 
Tabela 3. Relação dos ésteres do $\alpha$-tocoferol encontrados em plantas da Amazônia. Os ácidos graxos variam de $\mathrm{C}_{2}$ até $\mathrm{C}_{20}$. As séries dos tocoferóis beta, gama e delta estão representadas nos fragmentogramas das Figuras 5 e 6

\begin{tabular}{ccccccll}
\hline $\begin{array}{c}\mathrm{t}_{\mathrm{R}}(\min ) \\
\text { and }\end{array}$ & $\begin{array}{c}\mathrm{t}_{\mathrm{R}}(\min ) \\
\text { cup }\end{array}$ & $\begin{array}{c}\mathrm{t}_{\mathrm{R}}(\min ) \\
\mathrm{mar}\end{array}$ & $\begin{array}{c}\mathrm{t}_{\mathrm{R}}(\min ) \\
\text { cac }\end{array}$ & $\begin{array}{c}\mathrm{t}_{\mathrm{R}}(\min ) \\
\text { ser }\end{array}$ & $\begin{array}{c}\mathrm{t}_{\mathrm{R}}(\min ) \\
\text { cap }\end{array}$ & Compostos caracterizados & $\begin{array}{l}\text { Ions principais } \\
m / z\end{array}$ \\
\hline 25,1 & 25,1 & 22,6 & 25,0 & 25,1 & 25,3 & $\alpha$-tocoferol & 430,165 \\
nd & nd & nd & 25,3 & nd & nd & Acetato de $\alpha$-tocoferila & $472(430,165)$ \\
29,3 & nd & nd & nd & nd & 28,9 & Octanoato de $\alpha$-tocoferila & $556(430,165)$ \\
30,2 & nd & nd & nd & nd & 30,0 & Decanoato de $\alpha$-tocoferila & $584(430,165)$ \\
31,1 & 30,9 & 28,7 & 30,2 & nd & 31,1 & Dodecanoato de $\alpha$-tocoferila & $612(430,165)$ \\
nd & nd & nd & nd & nd & 31,6 & Tridecanoato de $\alpha$-tocoferila & $626(430,165)$ \\
32,3 & 31,9 & 29,6 & 31,0 & 32,0 & 32,1 & Tetradecanoato de $\alpha$-tocoferila & $640(430,165)$ \\
32,9 & 32,4 & nd & 31,4 & 32,5 & 32,6 & Pentadecanoato de $\alpha$-tocoferila & $654(430,165)$ \\
33,3 & 32,9 & 30,6 & 31,8 & 32,9 & 33,0 & Hexadecanoato de $\alpha$-tocoferila & $668(430,165)$ \\
nd & nd & nd & 32,2 & nd & 33,5 & Heptadecanoato de $\alpha$-tocoferila & $682(430,165)$ \\
34,3 & 33,8 & 31,6 & 32,6 & 33,8 & 33,9 & Octadecanoato de $\alpha$-tocoferila & $696(430,165)$ \\
34,8 & nd & nd & nd & nd & nd & Nonadecanoato de $\alpha$-tocoferila & $710(430,165)$ \\
35,3 & nd & nd & nd & nd & nd & Eicosanoato de $\alpha$-tocoferila & $724(430,165)$
\end{tabular}

and = andiroba; cup = cupuaçu; mar = marupá; cac = cacau; ser = seringueira; cap = caapi; nd = não detectado.

\section{CONCLUSÃO}

Neste trabalho foi possível observar que CGAR-AT-EM mostrou-se uma ferramenta excepcional na análise de compostos naturais, principalmente àqueles com massas elevadas, conforme já relataram Pereira e Aquino Neto ${ }^{1,2}$. Foi determinada a presença de ésteres graxos (ésteres de álcoois e ácidos graxos) em todas as amostras analisadas, com variações qualitativas e quantitativas de espécie para espécie, bem como ésteres graxos do fitol e de tocoferóis, sendo que esses últimos, a identificação foi confirmada por outros métodos (RMN ${ }^{13} \mathrm{C}$ e ${ }^{1} \mathrm{H}$; EM-AR).

\section{REFERÊNCIAS}

1. Pereira, A. S.; Aquino Neto, F. R.; Quim. Nova 2000, 3, 370.

2. Pereira, A. S.; Aquino Neto, F. R.; Trends Anal. Chem. 1999, 18, 126.

3. Elias, V. O.; Simoneit, B. R. T.; Pereira, A. S.; Cardoso, J. N.; J. Mass Spectrom. 1997, 32, 1356.

4. Pereira, A. S.; Silva, J. F. M.; Kiltzke, R.; Cardoso, J. N.; Aquino Neto, F. R.; Z. Naturfosch. 1999, 54c, 1115.

5. Siqueira, D. S.; Pereira, A. S.; Cabral, J. A.; Ferreira, C. A. C.; Aquino Neto, F. R.; Z. Naturfosch. 2000, 55c, 175
6. Post-Beittemiller, D.; Anna Ver. Plant Physiol. Plant Mol. Biol. 1996, 47, 405.

7. Reiter, B.; Lechner, M.; Lorbeer, E.; Aichholz, R.; J. High Resol. Chromatogr. 1999, 9, 514

8. Logan, B. A.; Monson, R. K.; Potosnak, M. J.; Trends Plant Sci. 2000, $11,477$.

9. Langenheim, J. H.; J. Chem. Ecol. 1994, 6, 1223.

10. Elias, V. O.; Simoneit, B. R. T.; Pereira, A. S.; Cabral, J. A.; Cardoso, J. N.; Environ. Sci. Technol. 1999, 14, 2369.

11. Rasool, N.; Ahmad, V. U.; Malik, A.; Phytochemistry 1991, 4, 1331.

12. Hasan, M.; Burdi, D. K.; Ahmad, V. U.; J. Nat. Prod. 1991, 5, 1444.

13. Griffiths, D. W.; Robertson, G. W.; Shepherd, T.; Ramsay, G.; Phytochemistry 1999, 52, 607.

14. Freedman, J. E.; Farhat, J. H.; Loscalzo, J.; Keaney Jr., J. F.; Circulation 1996, 10, 2434.

15. Dreher, F.; Gabard, B.; Schwindt, D.A.; Maibach, H. I.; Br. J. Derm. 1998 $2,332$.

16. Jacob, R. A.; Burri, B. J.; Am. J. Clin. Nutr. 1996, 6, $985 \mathrm{~S}$.

17. Palace, V. P.; Khaper, N.; Qin Q. I.; Singal, P. K.; Free Radic. Biol. Med. 1999, 5-6, 746.

18. Porter, Q. N.; Baldas, J.; Mass Spectrometry of Heterocyclic Compounds; Wiley-Interscience: New York, 1971, p. 99.

19. Pereira, A. S.; Siqueira, D. S.; Elias, V. O.; Simoneit, B. R. T.; Cabral, J. A.; Aquino Neto, F. R.; Phytochemistry 2002, 61, 711. 